\title{
Du travail des médecins et autres
}

\section{Eberhard Wolff}

PD Dr rer. soc., rédacteur culture, histoire, société

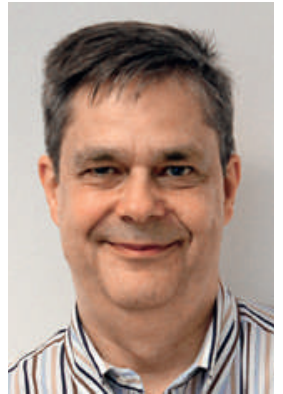

Cette publication nous rappelle régulièrement que le monde du travail médical (des soins ambulatoires) est en train de changer: plus de temps partiels, charge de travail allégée, moins de cabinets individuels [1, 2]. Le phénomène est-il conforme ou contraire à la tendance dans le monde du travail en général? Un nouveau recueil des Archives Sociales Suisses au titre ambigu, Ausser Betrieb (hors service/entreprise) [3], aide à répondre à cette question.

Dans leur introduction passionnante, les éditeurs, Brigitta Bernet et Jakob Tanner, notent que «l'activité professionnelle normale», réglementée par un contrat collectif, des employés qualifiés de l'industrie de l'époque du miracle économique fait progressivement place à des rapports de travail plus souples. Au lieu d'un travail à temps complet dans la même entreprise,

\section{Les médecins aussi se sentent aujourd'hui moins en phase avec leur profession, leur travail, leur cabinet.}

les contrats à durée déterminée, les temps partiels ou la (pseudo-)indépendance avec une disponibilité permanente ou dans plusieurs emplois se multiplient. Entrecoupés de pauses ou de périodes de chômage. Bref, nous travaillons de façon plus souple et plus incertaine, peut-être moins quantitativement mais de manière moins cloisonnée. Parce que nous le voulons, mais aussi parce que nous le devons.

La situation de départ est différente pour les médecins. En tant que petits entrepreneurs, ceux qui se sont établis ne font guère l'expérience de «l'activité professionnelle normale» réglementée. Et les médecins hospitaliers salariés ont accédé avec retard et seulement en partie au monde du travail régi par un contrat collectif de l'époque du miracle économique. Il suffit de voir leurs heures de travail.

Une partie du corps médical suit pourtant la tendance générale. Le désir de passer un jour par semaine avec les enfants ou au jardin est aussi vif chez les médecins - surtout les femmes - que les informaticiens. Ces dernières années, nous nous sommes écartés de l'ancien modèle d'une "société du travail» orientée vers l'entreprise, selon Bernet et Tanner. Le courtier en bourse acharné au travail est désormais une curiosité plus qu'un modèle. Les médecins aussi se sentent aujourd'hui moins en phase avec leur profession, leur travail, leur cabinet. Travailler dans un cabinet collectif ou un réseau de médecins permet de consacrer davantage de temps à sa famille ou ses passions.

En même temps, il y a des exceptions à la tendance générale. Dans un monde du travail assoupli, les travailleurs doivent de plus en plus raisonner en «autoentrepreneurs", selon Bernet et Tanner. Les médecins établis ont longtemps été des sortes de "Moi SA». Ils sont toujours plus nombreux à vouloir raisonner moins en chefs d'entreprise (individuelle). Ils se font salarier par une «entreprise» de cabinet médical, fondent un cabinet collectif ou un réseau de médecins, devenant ainsi au moins une «Nous SA».

Les frontières entre vie professionnelle et privée s'estompent. Publicitaires ou assureurs ramènent bon gré mal gré leur travail à la maison sur leur PC portable ou leur smartphone. Il en résulte des libertés, mais également une omniprésence du travail - chez les médecins aussi, bien sûr. Ils sont toutefois de plus en plus nombreux à vouloir échapper à la disponibilité permanente du rôle classique du médecin de campagne, par ex., et à rechercher une «activité professionnelle normale». Suivent-ils la tendance à l'assouplissement du travail ou y dérogent-ils? Il me semble que les médecins décrits ici ont les yeux rivés sur les aspects "vouloir» du monde du travail, assoupli et traditionnel. Ils cherchent des horaires réguliers, bien définis, mais suffisamment souples pour répondre à leurs souhaits individuels. Du fait de la pénurie de médecins, ils échappent pour l'instant sans doute plus facilement aux désagréables aspects «devoir» de l'actuel monde du travail assoupli, comme l'insécurité professionnelle et la dépendance. Je présume qu'il va se dégager un nouveau type (plus féminin?) de médecins, dont la conception du métier différera tout autant du médecin de campagne traditionnel que du (futur) médecin-chef, qui ont tous deux des horaires imposés par des tiers. Non pas «hors entreprise», mais hors des rôles classiques des médecins. J'en connais quelques-uns qui s'y emploient et d'autres qui en rêvent.

\section{Références}

1 Maurer L. Löst die Gemeinschaftspraxis die klassische Einzelpraxis ab? Bulletin des médecins suisses. 2014;95(25):971-2.

2 Hostettler S, Kraft E. Augmentation de la part de femmes et de médecins étrangers. Bulletin des médecins suisses. 2015;96(13):462-9.

3 Bernet B, Tanner J (éditeurs). Ausser Betrieb. Metamorphosen der Arbeit in der Schweiz. Zurich: Limmat-Verlag; 2015. 